\title{
Feeder-free maintenance of hESCs in mesenchymal stem cell-conditioned media: distinct requirements for TGF- $\beta$ and IGF-II
}

Rosa Montes ${ }^{1, *}$, Gertrudis Ligero ${ }^{1, *}$, Laura Sanchez ${ }^{1, *}$, Purificación Catalina ${ }^{1}$, Teresa de la Cueva ${ }^{1}$, Ana Nieto ${ }^{1}$, Gustavo J Melen ${ }^{1}$, Ruth Rubio ${ }^{1}$, Javier García-Castro ${ }^{1}$, Clara Bueno ${ }^{1}$, Pablo Menendez ${ }^{1}$

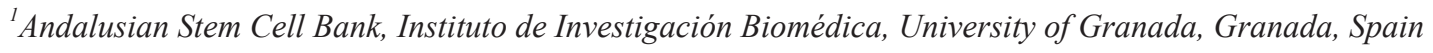

A paracrine regulation was recently proposed in human embryonic stem cells (hESCs) grown in mouse embryonic fibroblast (MEF)-conditioned media (MEF-CM), where hESCs spontaneously differentiate into autologous fibroblastlike cells to maintain culture homeostasis by producing TGF- $\beta$ and insulin-like growth factor-II (IGF-II) in response to basic fibroblast growth factor (bFGF). Although the importance of TGF- $\beta$ family members in the maintenance of pluripotency of hESCs is widely established, very little is known about the role of IGF-II. In order to ease hESC culture conditions and to reduce xenogenic components, we sought (i) to determine whether hESCs can be maintained stable and pluripotent using CM from human foreskin fibroblasts (HFFs) and human mesenchymal stem cells (hMSCs) rather than MEF-CM, and (ii) to analyze whether the cooperation of bFGF with TGF- $\beta$ and IGF-II to maintain hESCs in MEF-CM may be extrapolated to hESCs maintained in allogeneic mesenchymal stem cell (MSC)-CM and HFF-CM. We found that MSCs and HFFs express all FGF receptors (FGFR1-4) and specifically produce TGF- $\beta$ in response to bFGF. However, HFFs but not MSCs secrete IGF-II. Despite the absence of IGF-II in MSC-CM, hESC pluripotency and culture homeostasis were successfully maintained in MSC-CM for over 37 passages. Human ESCs derived on MSCs and hESCs maintained in MSC-CM retained hESC morphology, euploidy, expression of surface markers and transcription factors linked to pluripotency and displayed in vitro and in vivo multilineage developmental potential, suggesting that IGF-II may be dispensable for hESC pluripotency. In fact, IGF-II blocking had no effect on the homeostasis of hESC cultures maintained either on HFF-CM or on MSC-CM. These data indicate that hESCs are successfully maintained feeder-free with IGF-II-lacking MSC-CM, and that the previously proposed paracrine mechanism by which bFGF cooperates with TGF- $\beta$ and IGF-II in the maintenance of hESCs in MEF-CM may not be fully extrapolated to hESCs maintained in CM from human MSCs.

Keywords: TGF- $\beta$, IGF-II, bFGF, human ESCs, mesenchymal stem cells, conditioned media, feeder-free Cell Research (2009) 19:698-709. doi: 10.1038/cr.2009.35; published online 24 March 2009

\section{Introduction}

Human embryonic stem cells (hESCs) are pluripotent stem cells derived from human blastocyst-stage embryos [1]. Human ESCs are defined by both robust self-renewal

*These authors contributed equally to this work. Correspondence: Clara Bueno ${ }^{\mathrm{a}}$, Pablo Menendez ${ }^{\mathrm{b}}$ Tel: +34-958-894-672

a'E-mail: clara.bueno.exts@juntadeandalucia.es

bE-mail: pablo.menendez@juntadeandalucia.es

Received 5 October 2008; revised 28 October 2008; accepted 30 December 2008; published online 24 March 2009 capacity and pluripotent developmental potential in vitro and in vivo [1-4]. These distinctive properties of hESCs are not autonomously achieved, and recent evidence points to a level of external control from the microenvironment, through cell-cell interactions and soluble extrinsic factors $[5,6]$. Human ESCs are cultured in either feeder-based or feeder-free systems. Despite the fact that mouse embryonic fibroblasts (MEFs) are the most commonly used feeders for hESC co-culture [1], human feeders including human foreskin fibroblasts (HFFs) [7, 8] and human mesenchymal cells [9] have also been successfully explored in order to reduce xeno-components.

Among the feeder-free systems, MEF-conditioned 
media (MEF-CM) are the most widely employed [10, 11]. Moreover, more defined culture conditions such as serum replacement media containing high concentrations of basic fibroblast growth factor (bFGF) [12] or other exogenous factors such as TGF- $\beta$, Activin A, Noggin, insulin-like growth factor-II (IGF-II) or the Wnt agonist BIO among others have also been reported to be essential for undifferentiated growth of hESCs [reviewed in 13]. Despite the fact that hESCs have been successfully maintained on human fibroblasts [7, 8] and mesenchymal cells [9], only in recent work hESCs have been maintained in human fibroblast-CM [14]. However, to date no study has attempted to maintain hESCs in mesenchymal stem cell (MSC)-CM.

Recently, several members of the TGF- $\beta$ family of signalling molecules (Activin, Nodal and TGF- $\beta$ ) and IGFII have been shown to be necessary for the maintenance of pluripotency in hESCs [5, 15-18]. Importantly, despite the evolution of the hESC culture conditions and the finding of these several key factors, the supplementation with bFGF to sustain hESC potential is still required. In fact, both TGF- $\beta$ and IGF-II have been proposed to cooperate with the FGF pathway to maintain feeder-free hESC cultures in xenogenic MEF-CM by establishing a regulatory stem cell niche $[5,18]$. Thus, a model of paracrine regulation was recently proposed [5] within feederfree hESC cultures maintained in MEF-CM, where hESC colonies spontaneously differentiate into hESC-derived fibroblast-like cells (hdFs) to maintain culture homeostasis. These autologous hdFs support hESC culture homeostasis by releasing, in response to bFGF, the hESC supportive factors TGF- $\beta$ and IGF-II, which have a direct role in the survival and self-renewal of hESCs [5].

In order to facilitate hESC culture conditions and to reduce xenogenic components, we sought to determine whether different hESC lines can be maintained stable and sustain pluripotency in a feeder-free system using $\mathrm{CM}$ from human feeders such as MSCs and HFFs rather than xenogeneic MEF-CM. Additionally, we aimed at establishing whether the previously proposed cooperation of exogenous bFGF with TGF- $\beta$ and IGF-II to maintain hESCs in MEF-CM may be extrapolated to hESC cultures maintained in CM from allogeneic human feeders.

We found that MSCs and HFFs express the four putative FGF receptors (FGFR1-4) and specifically produce TGF- $\beta$ in response to bFGF. However, HFFs but not MSCs secrete IGF-II. Despite the absence of IGF-II in MSC-CM, hESC pluripotency and culture homeostasis were successfully maintained in MSC-CM beyond 23 passages. Similar to HFF-CM, hESCs derived on MSCs and those maintained in MSC-CM retained typical hESC morphology, euploidy, expression of pluripotency-asso- ciated surface markers and transcription factors and displayed in vitro and in vivo differentiation potential into tissues representing mesoderm, ectoderm and endoderm. These data suggest that hESCs can be successfully maintained feeder-free in IGF-II-lacking MSC-CM, and that the previously proposed paracrine mechanism by which bFGF cooperates with TGF- $\beta$ and IGF-II in the maintenance of hESCs in MEF-CM may not be fully extrapolated to hESCs maintained in CM from human feeders.

\section{Results}

TGF- $\beta$ is secreted by MSCs and HFFs, and its production increases in response to $b F G F$

Many efforts have been made to develop hESC culture systems based on the use of human feeders $[8,9,19$ 24]. However, among the feeder-free culture systems, the most widely established is still based on the use of xenogenic MEF-CM. Only in very recent work have hESCs been maintained feeder-free using human fibroblast-CM [14]. To date no study has analyzed whether hESCs may be successfully maintained in MSC-CM. We therefore sought to determine whether hESCs can be maintained pluripotent and feeder-free in MSC-CM and HFF-CM.

We initially analyzed whether allogeneic human mesenchymal stem cells (hMSCs) and HFFs used to produce $\mathrm{CM}$ of human origin release TGF- $\beta$ and IGF-II in response to bFGF as recently reported for autologous hdFs [5]. Accordingly, the expression of all known putative FGF receptors was analyzed in both proliferating hMSCs (Figure 1A) and HFFs (Figure 1C). RT-PCR analysis revealed that both hMSCs (Figure 1B) and HFFs (Figure 1D) expressed the four FGF receptors (FGFR1-4). Next, we determined whether TGF- $\beta$, a well-established hESCsupportive factor that cooperates with bFGF, is produced by these human feeders in response to exogenous bFGF $[5,15,18]$. Human TGF- $\beta$ was readily detectable at low levels in MSC-CM (Figure 1E) and HFF-CM (Figure 1F). Importantly, its concentration significantly increased in response to exogenous bFGF: 3.6-fold increase in MSC-CM (Figure 1E) and 5.8-fold increase in HFF-CM (Figure $1 \mathrm{~F}$ ). To further confirm TGF- $\beta$ production in response to bFGF, MSC-CM and HFF-CM were independently harvested on day 2 (1st harvest) and day 5 (2nd harvest) after cell seeding and a reproducible trend was observed for both hMSCs and HFFs, which clearly produced additional TGF- $\beta$ upon bFGF exposure regardless of the time point of $\mathrm{CM}$ harvesting. Chemical inhibition of FGFR1 prior to supplementation of bFGF reduced the release of TGF- $\beta$ by $36 \%$ in HFFs and $62 \%$ in MSCs (Figure $1 \mathrm{G}$ ), demonstrating that this functional cooperation between bFGF and TGF- $\beta$ seems specific. Together, 
A

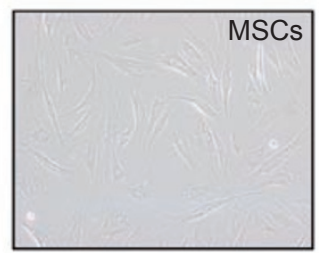

C

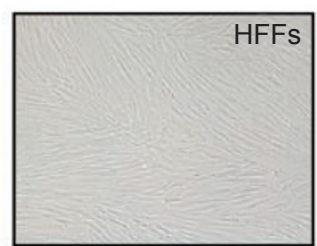

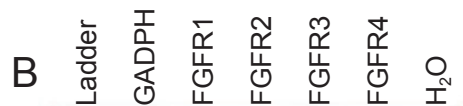

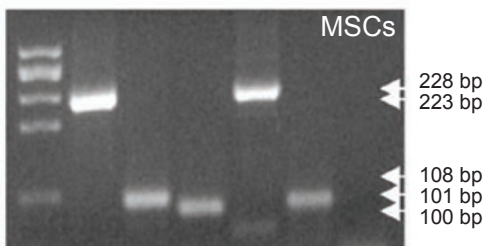

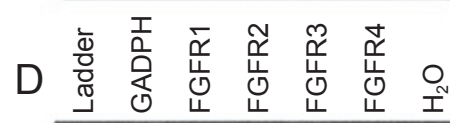

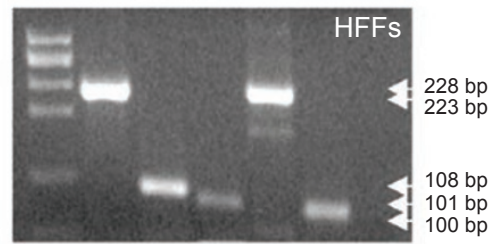

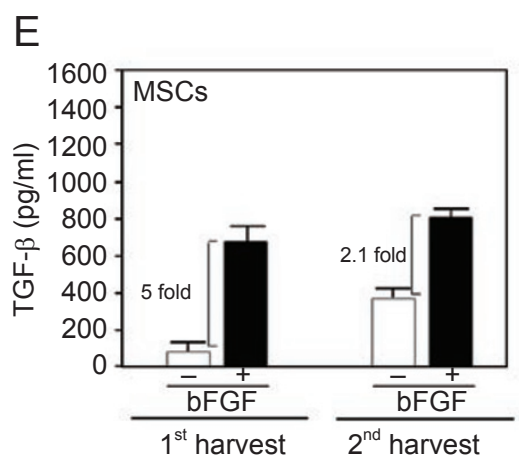

$\mathrm{F}$

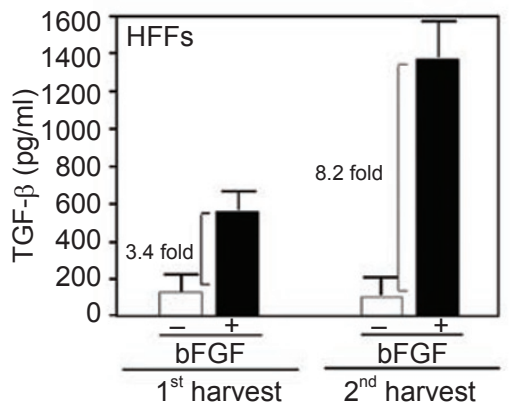

G

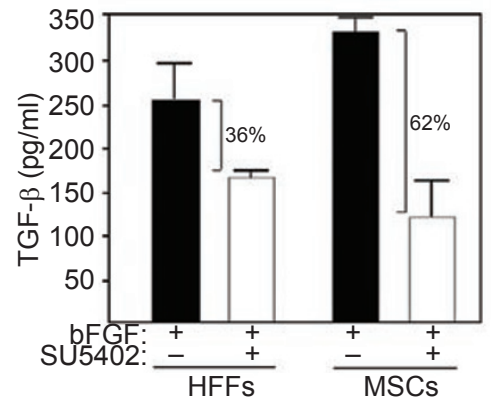

Figure 1 TGF- $\beta$ is produced in response to bFGF by MSCs and HFFs. Phase-contrast morphology of MSCs (A) and HFFs (C). RT-PCR showing mRNA expression of FGFR1-4 in MSCs (B) and HFFs (D). Amount of TGF- $\beta$ produced by MSCs (E) and HFFs (F) in response to bFGF. (G) Specific blockage of TGF- $\beta$ production by the FGFR chemical inhibitor SU5402.

these results indicate that similar to autologous hdFs, allogeneic hMSCs and HFFs produce TGF- $\beta$ in response to exogenous bFGF.

HFFs but not MSCs secrete IGF-II, and the production of IGF-II is not augmented in response to bFGF in either human-derived feeders

Still very little is known about the role of IGF-II in hESC pluripotency. IGF-II has been implicated in clonal outgrowth of hematopoietic stem cells [25] and IGFII and IGFR are expressed in both mouse and human blastocysts [26]. Moreover, a paracrine mechanism by which bFGF cooperates not only with TGF- $\beta$ but also with IGF-II to maintain feeder-free hESCs in MEF-CM was recently reported for the first time [5]. We therefore sought to determine whether this functional cooperation between bFGF and IGF-II to maintain hESCs in MEF$\mathrm{CM}$ may be extrapolated to hESC cultures maintained in
MSC-CM/HFF-CM. In line with previous data [5], we first confirmed that IGF-II is undetectable in MEF-CM and basal hESC media but that it is detectable in media conditioned by autologous hdFs differentiated from hESCs (data not shown). Interestingly, although HFFs produce significant basal levels of IGF-II $(2800 \pm 260 \mathrm{pg} /$ $\mathrm{ml})$, IGF-II concentration does not increase in response to exogenous bFGF (Figure 2A). Human MSCs, however, do not secrete IGF-II prior to or after exposure to bFGF (Figure 2A). To confirm the ELISA data, we determined by real-time RT-PCR the mRNA expression level of IGF-II in HFFs and hMSCs in the presence or absence of bFGF. In line with the ELISA results, HFFs showed high expression of IGF-II mRNA (Figure 2B). The IGFII mRNA expression was even higher than that observed in the MCF7 cell line used as positive control ( $C t$ values: $20 v s$ 26, respectively). As expected, exogenous bFGF did not change the IGF-II mRNA expression (Figure 2B; 
A

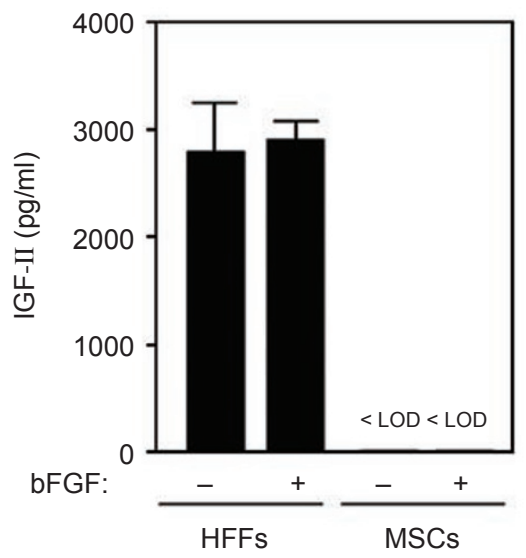

B
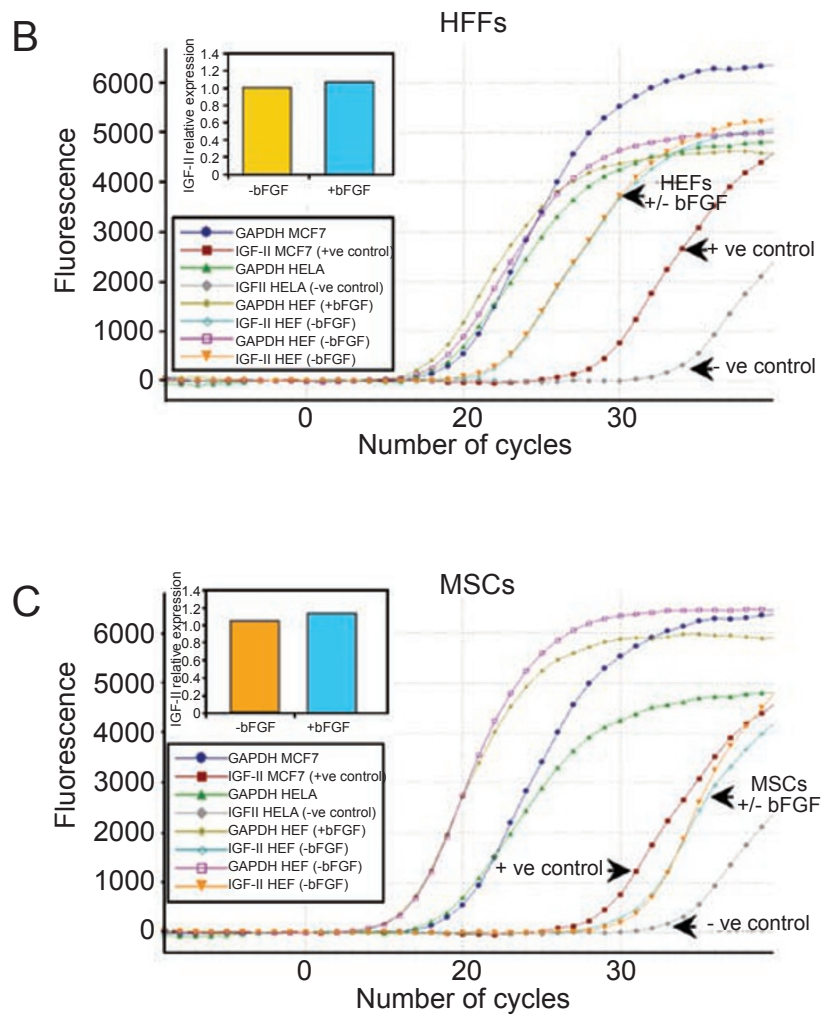

Figure 2 IGF-II production is not altered in response to bFGF by either MSCs or HFFs. (A) Levels of soluble IGF-II produced by HFFs and MSCs in the presence or absence of bFGF. In HFFs soluble IGF-II is readily detectable but its concentration does not increase in response to bFGF. In contrast, MSCs do not produce IGF-II. LOD=limit of detection. (B, C) Representative real-time RTPCR confirming the absence of IGF-II in MSC-CM observed in the ELISA. (B) HFFs expressed higher levels of IFG-II mRNA $\left(C_{t}=20\right)$ than the positive control (MCF-7 cell line). However, no differences were observed in the mRNA levels in the presence or absence of bFGF (inset). (C) According to the ELISA data, MSCs express low levels of mRNA for IGF-II $\left(C_{\mathrm{t}}=32\right)$. Similar to HFFs, the relative amounts of mRNA IGF-II was identical in the absence or presence of bFGF, together indicating that at least in these human feeders, bFGF cooperates with TGF- $\beta$ but not with IGF-II. inset panel). The real-time RT-PCR also confirmed the lack of IGF-II production by MSCs measured by ELISA (Figure 2C). As expected, hMSCs showed very low expression of IGF-II mRNA (Figure 2C) and the IGF-II mRNA expression remained low regardless of the presence or absence of bFGF (Figure 2C; inset panel). These data are consistent with previous reports suggesting that the distinct ability of mouse and human feeders in promoting undifferentiated growth of hESCs is attributable to their hESC-supportive growth factor production [20]: both HFFs and hMSCs produce TGF- $\beta$, whereas MSCs, in contrast to HFFs, are unable to release IGF-II.

Human ESCs retain in vitro and in vivo pluripotency in IGF-II-lacking MSC-CM

The analysis performed on MSC-CM and HFF-CM unravelled significant differences regarding the TGF- $\beta$ and IGF-II composition. HFF-CM contains both TGF- $\beta$ and IGF-II, while MSC-CM contains TGF- $\beta$ but lacks IGF-II. We then wanted to determine whether hESCs can be maintained stable and pluripotent using allogeneic MSC-CM and HFF-CM rather than MEF-CM, taking into account the differences in IGF-II production between the two human feeders.

Two hESC lines, HS181 and SHEF1, were cultured for 23-37 passages feeder-free either in MSC-CM or in HFF-CM. Despite the absence of IGF-II in MSC-CM, both $\mathrm{hESC}$ lines retained pluripotency and undifferentiated growth in MSC-CM as they did in HFF-CM, which contains both factors, TGF- $\beta$ and IGF-II. No differences in in vitro and in vivo pluripotency and culture homeostasis were observed among hESC lines maintained in MSC-CM versus HFF-CM. Identical to hESCs cultured in HFF-CM, those maintained in MSC-CM retained typical hESC morphology and culture homeostasis (Figure 3 ; left panels) and expression (analyzed by immunocytochemistry and flow cytometry) of the pluripotencyassociated surface markers SSEA-3, SSEA-4, Tra-160 and Tra-1-81 (Figure 3). Similarly, both hESC lines expressed the transcription factors Oct $3 / 4$, Nanog, Rex-1 and Sox-2 (Figure 4A) and remained karyotypically stable (Figure 4B). Functionally, both hESC lines successfully differentiated in vitro through EB formation (Figure 5A; left panel) into tissues representing the three germ layers: endoderm ( $\alpha$-fetoprotein + cells; Figure $5 \mathrm{~A})$, mesoderm (Actin+ cells; Figure 5A) and ectoderm ( $\beta$-IIITubulin+ cells; Figure 5A). The gold-standard pluripotency assay relies on the ability to form in vivo teratomas upon injection into immune-deficient mice. HS181 and SHEF1 hESC lines maintained either in MSC-CM or in HFF-CM formed teratomas 8-10 weeks after inoculation. These complex and disorganized tumours contained 


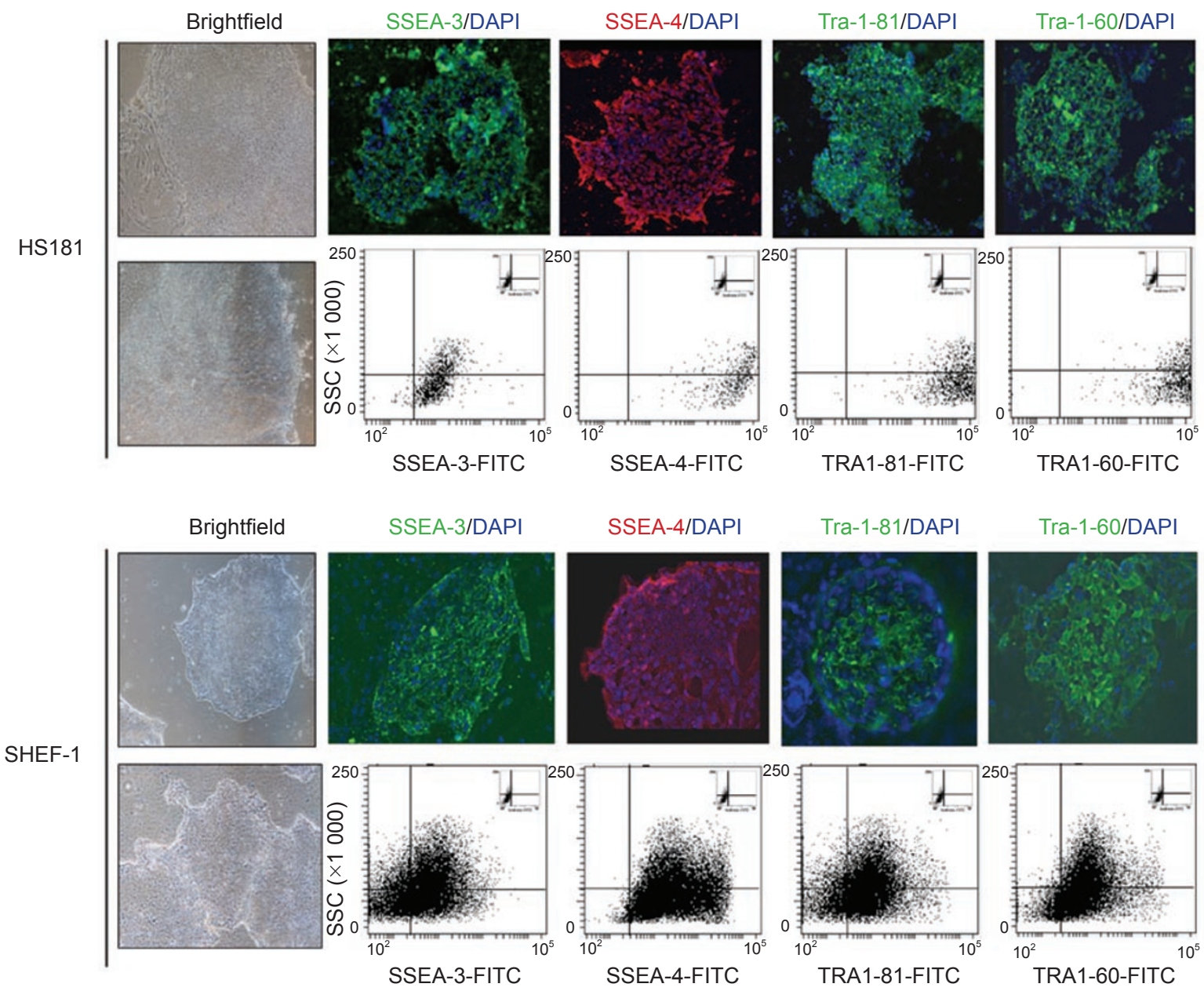

Figure 3 Representative immunophenotypic characterization of HS181 and SHFF1 hESC lines maintained feeder-free with MSC-CM or HFF-CM. Phase-contrast photomicrographs showing typical hESCs morphology (left panels). Expression of the pluripotency-associated surface markers SSEA-3, SSEA-4, Tra-1-60 and Tra-81 was detected both by immunocytochemistry (top row) and by flow cytometry (bottom row; insets represent isotype controls). For immunocytochemistry staining, colours are indicated. Nuclei were counterstained with 4,6-diamidino-2-phenylindole (DAPI, blue).

a variety of tissues representing the three germ layers (Figure 5B and 5C), thus demonstrating the pluripotent features of hESC lines, regardless of their growth in IGFII-lacking MSC-CM or IGF-II-containing HFF-CM.

Human ESC cultures maintained in MSC-CM displayed growth kinetics and doubling time similar to those maintained in HFF-CM. A slight proliferative disadvantage could be observed just during the first 2-3 passages for those hESCs grown in MSC-CM (Figure 6A). However, no differences in cellular death/apoptosis were observed between hESCs grown in either condition (Figure 6B), suggesting a slower culture adaptation for those hESCs grown in MSC-CM. These data indicate that hESCs are successfully maintained feeder-free in MSC-CM lacking IGF-II, suggesting that IGF-II may be dispensable for hESC pluripotency. Additionally, the cooperation of bFGF with TGF- $\beta$ and IGF-II in the maintenance of hESCs in MEF-CM might not be fully extrapolated to hESCs maintained in allogeneic MSCCM lacking IGF-II.

Distinct requirements of IGF-II for hESC maintenance in $M E F-C M$ versus allogeneic MSC-CM or HFF-CM

IGF-II was recently reported to be a candidate hESCsupportive factor [5], but its supplementation is not required in the absence of feeders to maintain hESCs in MEF-CM, suggesting endogenous IGF-II production in $\mathrm{hESC}$ cultures maintained in MEF-CM [5]. Accordingly, a paracrine regulation seems to occur in feederfree hESC cultures grown in MEF-CM, by which hESCs spontaneously differentiate into hdFs to maintain culture homeostasis by producing IGF-II in response to bFGF.

Here, we first confirmed that IGF-II is undetectable in MEF-CM and basal hESC media, but that its levels 


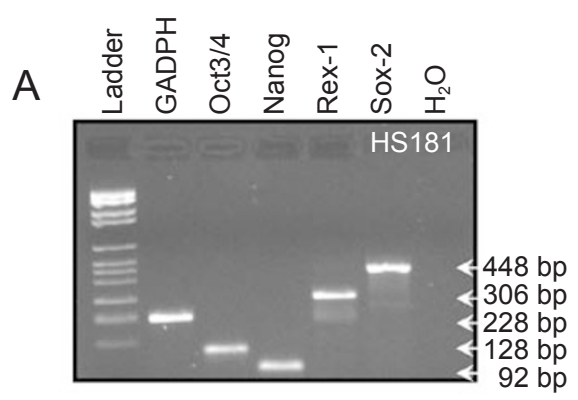

B

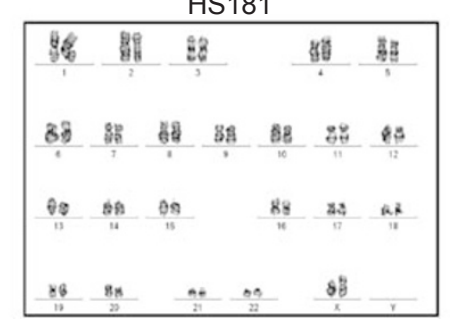

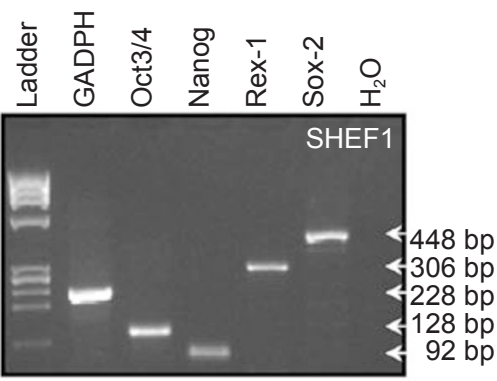

SHEF1

\begin{tabular}{|c|c|c|c|c|c|c|}
\hline 鮊 & 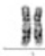 & & & & 解 & 些 \\
\hline 86 & 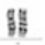 & ถ8 & $\mathbb{B E}$ & กำ & 83 & 38 \\
\hline da & tet & As & & $B$ & 88 & 93 \\
\hline if & 88 & & & & t &. \\
\hline
\end{tabular}

Figure 4 Molecular and cytogenetic characterization of HS181 and SHEF1 hESC lines maintained either with MSC-CM or with HFFCM. (A) RT-PCR analysis displaying mRNA expression of the undifferentiated markers Oct3/4, Nanog, Rex-1 and Sox-2. GAPDH was used as a housekeeping gene. (B) Conventional G-banding confirming karyotypic stability of hESCs maintained for 30 passages feeder-free in MSC-CM or HFF-CM.

A
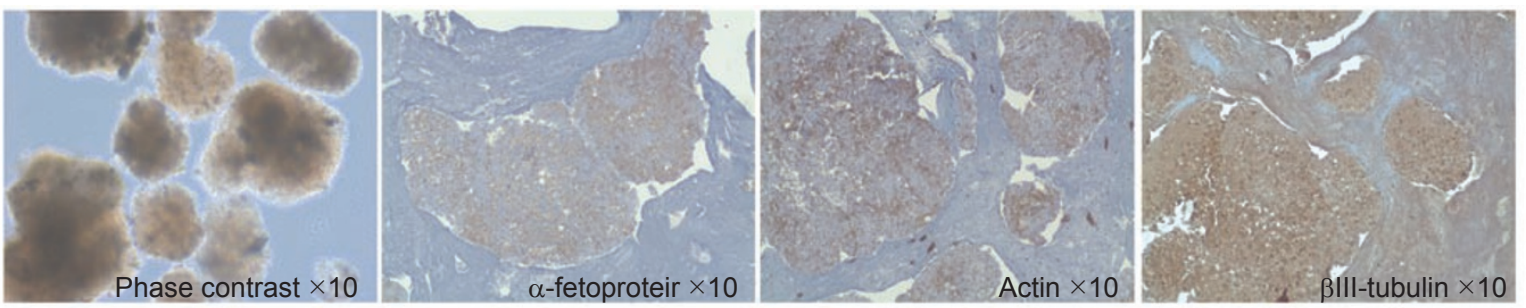

B
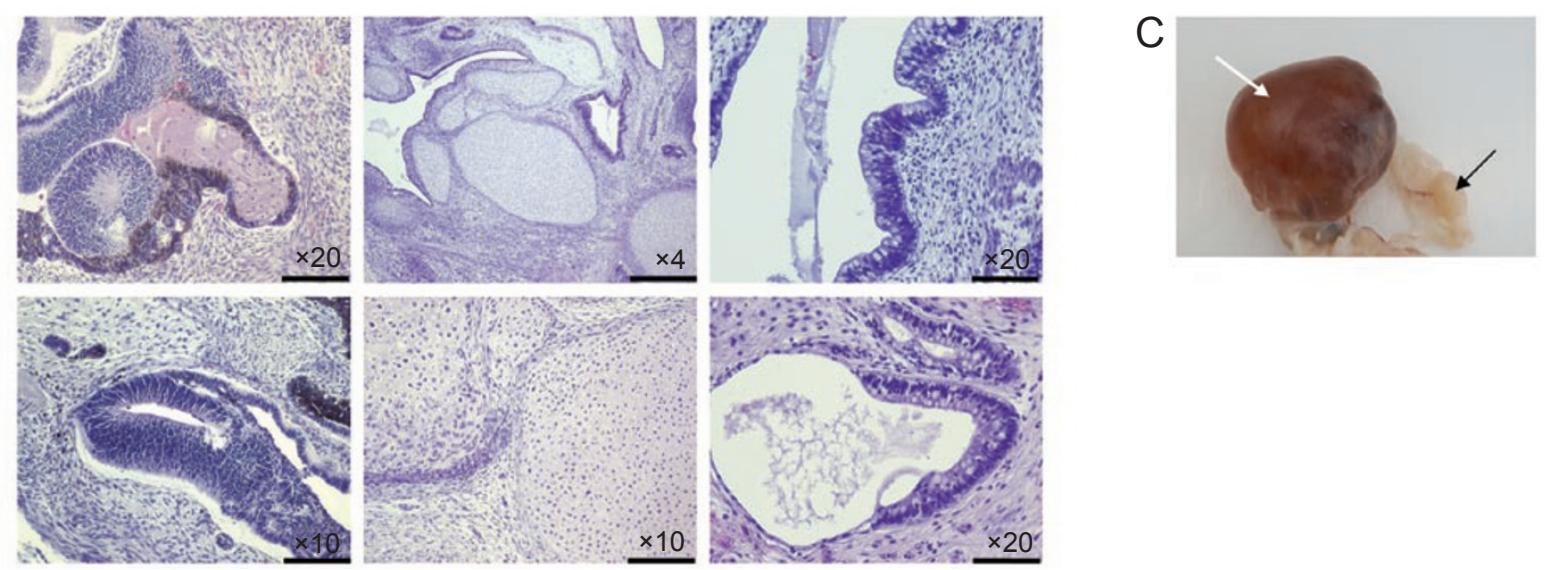

Figure 5 Representative images of in vitro and in vivo multi-lineage differentiation capacities of HS181 and SHEF1 hESC lines maintained feeder-free in MSC-CM or HFF-CM. (A) Phase-contrast (left panel) and immunohistochemistry images showing the ability of hESC lines to differentiate into tissues representing the three germ layers: endoderm ( $\alpha$-fetoprotein), mesoderm (actin) and ectoderm ( $\beta$ III-tubulin). (B) Human ESCs cultured either in MSC-CM or in HFF-CM form teratomas containing tissues representing the three germ layers. A representative hematoxylin-eosin histology of HS181 (top panels) and SHEF1 (bottom panels) shows primitive neural and epidermal epithelia (ectoderm-origin; left panels), cartilage (mesoderm-origin; middle panels) and cylindrical epithelial and goblet cells (endoderm-origin; right panels). (C) Representative macroscopic image of 8-weeks teratoma after injection of hESCs maintained in MSC-CM (white arrow). Note the size increase as compared to PBS-injected testis (black arrow). 
A
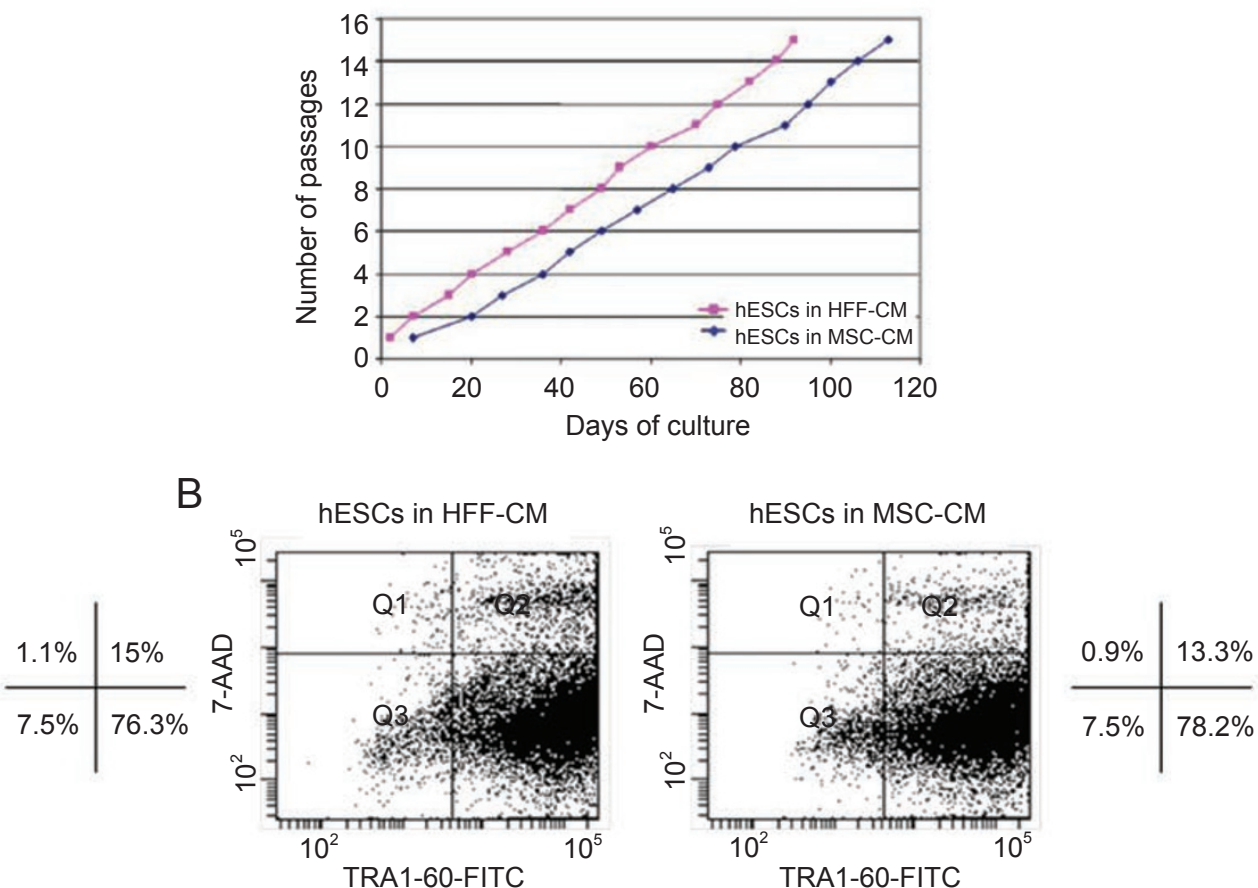

C

Human IGF-II (pg/mL)

\begin{tabular}{|c|c|c|c|c|}
\hline HFF-CM & $\begin{array}{c}\text { HFF-CM } \\
\text { hESC conditioned }\end{array}$ & MSC-CM & $\begin{array}{c}\text { MSC-CM } \\
\text { hESC conditioned }\end{array}$ & $\begin{array}{c}\text { hESC media conditioned by } \\
\text { hESC derived and co-cultured over hMSCs }\end{array}$ \\
\hline $6300 \pm 70$ & $3700 \pm 213$ & $<$ LOD & $<$ LOD & $<$ LOD \\
\hline
\end{tabular}

D

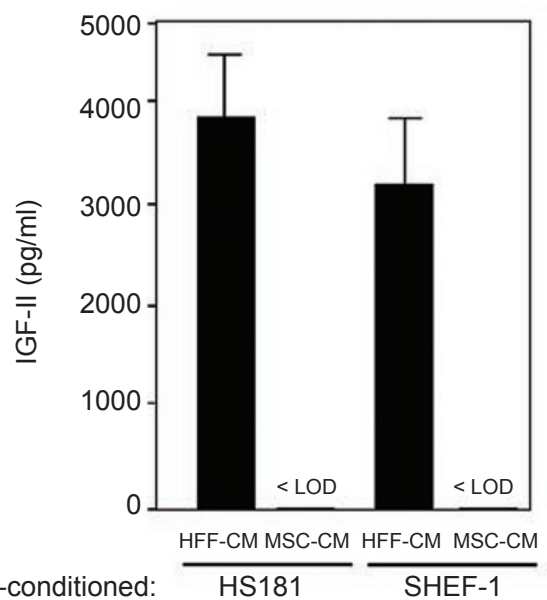

Figure 6 Growth and survival of hESCs maintained in MSC-CM versus HFF-CM and IGF-II concentration in hESC-conditioned MSC-CM or HFF-CM. (A) Cell growth curves of hESCs in MSC-CM (blue line) and in HFF-CM (red line). (B) Frequency of hESCs undergoing cell death/apoptosis in MSC-CM (right panel) and in HFF-CM (left panel) analyzed by flow cytometry after 7-AAD staining and/or Annexin-V staining. (C) Concentration of IGF-II in the indicated media. LOD: limit of detection. (D) Concentration of IGF-II detailed for HS181- and SHEF-1-conditioned MSC-CM or HFF-CM. LOD: limit of detection. 
increase, which are readily detectable in media exposed to autologous hdFs [5 and data not shown]. On the other hand, HFFs readily produce basal levels of IGF-II, with an average concentration in HFF-CM of $6300 \pm 70 \mathrm{pg} /$ $\mathrm{ml}$ (Figure 6C). However, in contrast to autologous hdFs, IGF-II concentration did not increase in response to bFGF measured by ELISA and Q-RT-PCR (Figure $2 \mathrm{~A}$ and $2 \mathrm{~B}$ ). Human MSCs do not secrete IGF-II either before or after exposure to bFGF (Figure 2A and 6C). Similar to HFFs, the very low basal expression of IGFII transcript in hMSCs was not increased in response to bFGF (Figure 2C). These data indicate that while autologous hdFs (either FACS-isolated or in vitro differentiated from hESCs) produce IGF-II in response to bFGF, human feeders such as HFFs and MSCs fail to do so. Moreover, despite clear differences in IGF-II content between HFF-CM and MSC-CM, hESC lines are equally maintained pluripotent in IGF-II-containing HFF-CM or IGF-II-lacking MSC-CM, suggesting that (i) autologous hdFs spontaneously differentiating from hESCs cultured in MSC-CM or HFF-CM, similar to what we have shown for hESCs maintained in MEF-CM, produce IGF-II, compensating its absence in the MSC-CM, or (ii) IGF-II is dispensable for maintenance of hESC pluripotency in hMSC-CM.

In order to test these two possibilities, the concentration of IGF-II was measured in IGF-II-containing HFF$\mathrm{CM}$ and IGF-II-lacking MSC-CM before and after exposure to hESC cultures (Figure 6C and 6D). Detectable amounts of IGF-II were found in hESC-conditioned HFF-CM. However, no IGF-II was detectable in MSC$\mathrm{CM}$ either prior to or after exposure to hESC cultures (Figure 6C and 6D). Furthermore, the undetectable levels of IGF-II in MSC-CM were reproducible after exposure to distinct hESC lines (HS181 and SHEF1) (Figure 6C and 6D). These data indicate that it is unlikely that hdFs derived from hESCs cultured in MSC-CM produced IGF-II, suggesting that MSC-CM might contain unknown factors interfering with the IGF-II production by hdFs. Alternatively, it remains plausible that allogeneic hMSCs produce other uncharacterized hESC-supportive factors, which make the presence of IGF-II irrelevant for hESC maintenance [20]. Overall, our data support that IGF-II may be dispensable for maintenance of hESC pluripotency in MSC-CM.

To gain further insights into the role of IGF-II in hESC derivation and maintenance, we measured the presence of IGF-II during the co-culture of a fully characterized hESC line recently derived in our laboratory on hMSCs (Cortes et al., submitted). Up until now, this hESC line (termed AND-1) has been successfully maintained on hMSCs with hESC basal media supplemented with $8 \mathrm{ng} /$ $\mathrm{ml}$ of $\mathrm{bFGF}$ beyond 30 passages, while displaying typical hESC morphology, euploidy, expression of pluripotencyassociated surface markers and transcription factors, and in vitro and in vivo multi-lineage developmental potential (Cortes et al., submitted). In line with the undetectable IGF-II levels measured by ELISA in MSC-CM and the very low IGF-II transcript expression in MSCs, no IGFII could be detected in hESC media conditioned by this $\mathrm{hESC}$ line derived on and maintained in a MSC co-culture (Figure 6C). The successful derivation, maintenance of pluripotency and culture homeostasis of this hESC line derived and grown on hMSCs, together with the absence of IGF-II in the media conditioned by this hESC line, further demonstrate that IGF-II is dispensable for the maintenance of hESCs feeder-free in MSC-CM or on MSCs feeder layer.

To further confirm that IGF-II is dispensible for the maintenance of hESCs in HFF-CM and MSC-CM and to exclude the possibility of IGF-II secretion from hESCs themselves, IGF-II was specifically blocked with an IGFII-neutralizing antibody. After 16 days, IGF-II blocking had no effects on the overall homeostasis of hESC cultures maintained either on HFF-CM (Figure 7A-7C) or on MSC-CM (Figure 7D-7F). Treatment with an IGFII-neutralizing antibody did not induce morphological changes (Figure 7A and 7D) and had little to no effect on cell proliferation, apoptosis, expression of the ESCassociated markers Tra-1-81 and SSEA-3 (Figure 7B and $7 \mathrm{E})$ and expression of the ESC-specific transcription factors Nanog, Oct3/4, Rex 1 and Sox 2 (Figure 7C and 7F).

\section{Discussion}

Many efforts have been made to develop hESC culture systems based on human feeders $[8,9,19,20,22-$ 24]. However, among the feeder-free culture systems, the most widely established is still based on the use of MEFCM. Only in very recent work have hESCs been maintained feeder-free using human fibroblast-CM [14]. No study has analyzed so far whether hESCs may be successfully maintained in MSC-CM. The rationale behind maintaining hESCs in MSC-CM or even deriving them on MSCs is two-fold: (i) to reduce the presence of xenocomponents, and (ii) because hESC maintained with MSC-CM or derived on MSCs might be more prone to differentiation toward mesoderm lineages.

We have recently shown that hESCs maintained in MEF-CM differentiate into autologous hdFs, which maintain culture homeostasis by producing TGF- $\beta$ and IGF-II in response to bFGF [5]. Very little is known about the role of IGF-II in the maintenance of hESC pluripotency. Here, we aimed at determining whether 
hESCs in HFF-CM

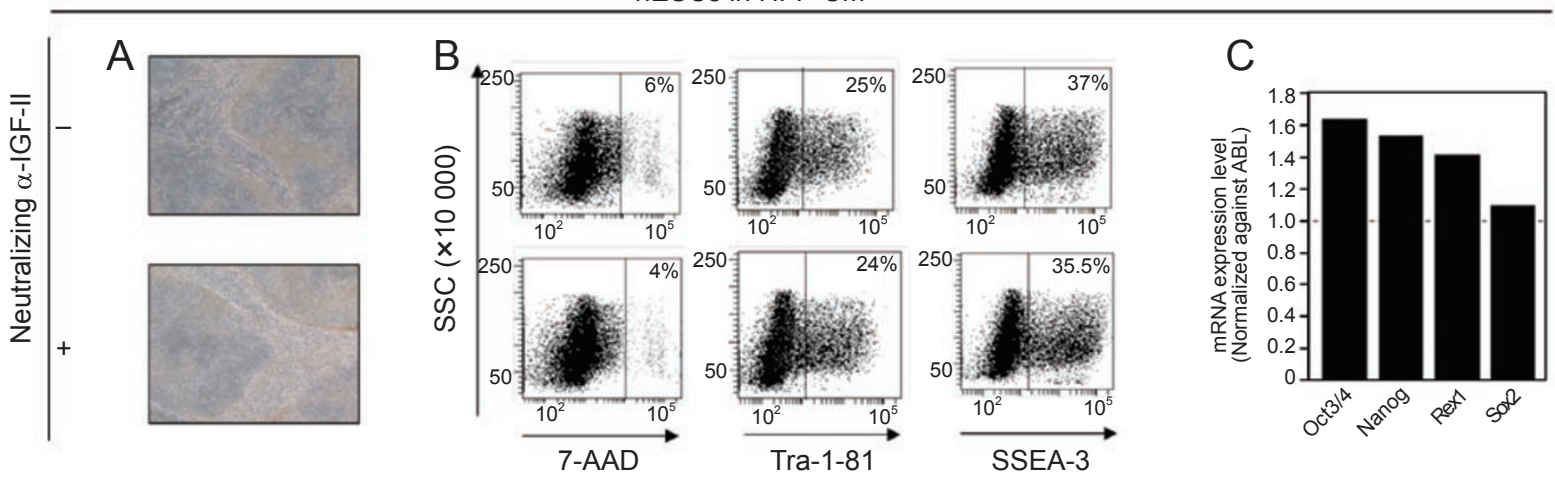

hESCs in MSC-CM
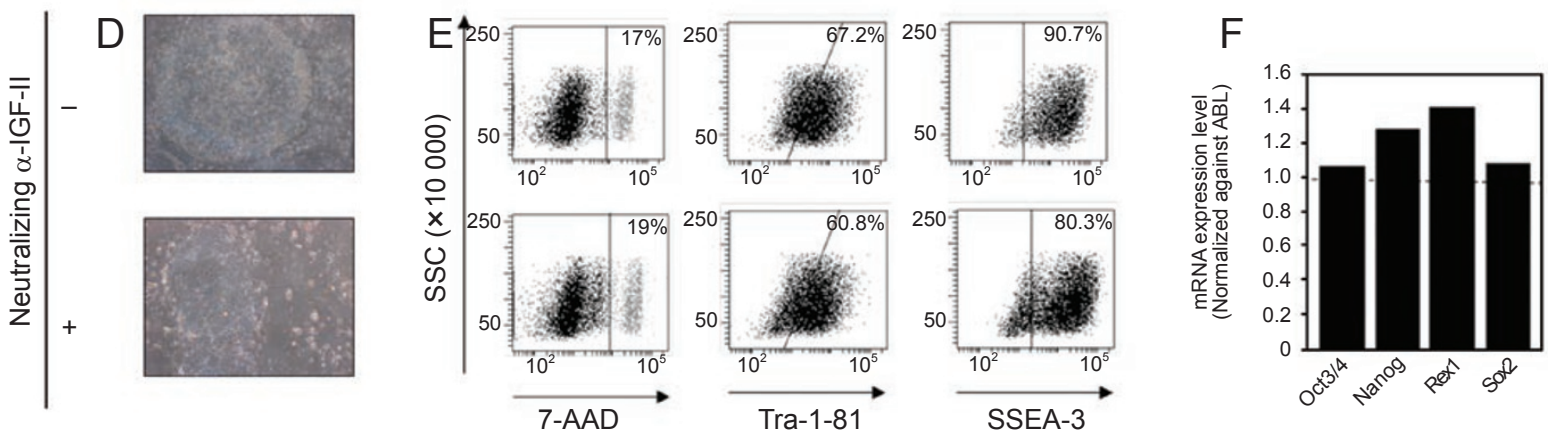

Figure 7 IGF-II blocking does not affect the homeostasis of hESCs maintained in HFF-CM or MSC-CM. Phase-contrast images of hESC cultures maintained in HFF-CM (A) or MSC-CM (D) with or without the IGF-II neutralizing antibody. 7-AAD staining and expression of Tra-1-81 and SSEA-3 in hESC cultures maintained in HFF-CM (B) or MSC-CM (E) with or without the neutralizing $\alpha-I G F-I I$. Oct3/4, Nanog, Rex1 and Sox2 transcript expression in hESCs maintained in HFF-CM (C) or MSC$\mathrm{CM}(\mathrm{F})$ in the presence of the neutralizing $\alpha-I G F-I I$. The mRNA expression is relative to that from hESCs maintained in the absence of $\alpha-I G F-I I$ and normalized against the indicated housekeeping gene.

hESCs can be maintained stable and pluripotent using allogeneic MSC-CM and HFF-CM rather than xenogenic MEF-CM, and to analyze whether the cooperation of bFGF with TGF- $\beta$ and IGF-II in MEF-CM may be extrapolated to hESCs maintained in allogeneic MSC-CM and HFF-CM.

Similar to feeder-free culture in MEF-CM, both MSCs and HFFs specifically produce TGF- $\beta$, a well-established hESC-supportive factor, in response to exogenous bFGF. As previously reported [5], IGF-II was undetectable in MEF-CM and basal hESC media but was detectable in media conditioned by autologous hdFs differentiated from hESCs. Interestingly, HFFs readily produce IGFII whereas MSCs do not secrete IGF-II at all, and the production of IGF-II is not induced in response to bFGF in either human feeder. Our data reveal differences in the composition of TGF- $\beta$ and IGF-II factors between HFF$\mathrm{CM}$ and MSC-CM and confirm that the distinct ability of mouse and human feeders to promote undifferentiated
hESC growth may be attributable to their hESC-supportive growth factor production [20].

Despite the significant differences regarding the composition of IGF-II between HFF-CM and MSC-CM, several hESC lines can be equally maintained stable and pluripotent for over 37 passages using allogeneic MSC$\mathrm{CM}$ and HFF-CM. Human ESCs derived on MSCs and those maintained in MSC-CM retained typical morphology, euploidy, expression of surface markers and transcription factors associated with pluripotency and displayed in vitro and in vivo multilineage developmental potential. Human ESCs maintained in MSC-CM displayed during the first 2-3 passages slower growth as compared to hESCs grown in HFF-CM. Due to the lack of differences in doubling time and apoptosis between hESCs grown in MSC-CM and those grown in HFF-CM, it may be plausible that rather than a proliferative disadvantage, a delayed culture adaptation of hESCs grown in MSC-CM occurred. Thus, hESCs are successfully main- 
tained feeder-free in MSC-CM lacking IGF-II, suggesting that IGF-II may be dispensable for hESC pluripotency. The functional cooperation of bFGF with TGF- $\beta$ and IGF-II reported for hESCs in MEF-CM might not be extrapolated to hESCs maintained in allogeneic IGF-IIlacking MSC-CM.

While autologous hdFs produce IGF-II in response to bFGF, allogeneic human feeders fail to do so. Moreover, despite clear differences in the IGF-II content between HFF-CM and MSC-CM, hESC lines are equally maintained pluripotent in IGF-II-containing HFF-CM or IGFII-lacking MSC-CM, suggesting that (i) autologous hdFs derived from hESCs cultured in MSC-CM or HFF-CM produce IGF-II, thus compensating its absence in the MSC-CM or (ii) IGF-II is dispensable for maintenance of hESC pluripotency in human feeders-derived CM. To distinguish between these two possibilities, the concentrations of IGF-II in HFF-CM and MSC-CM before and after exposure to hESC cultures were measured. hdFs derived from hESCs cultured in MEF-CM produced IGFII [5], whereas those derived from hESCs cultured in allogeneic HFF-CM or MSC-CM failed to produce IGFII, suggesting that allogeneic human-derived CM might contain unknown factors interfering with IGF-II production by hdFs. Alternatively, it remains plausible that human feeders produce alternative uncharacterized hESCsupportive factors, which make the presence of IGF-II irrelevant for hESC maintenance [20]. Two-dimensional protein fractionation experiments are being considered in our laboratory in order to facilitate a comprehensive mapping of soluble protein profiles in distinct mouseand human-derived CM. Overall, our data support that IGF-II may be dispensable for maintenance of hESCs in human feeder-derived CM, rather than the hypothesis that autologous hdFs differentiated from hESCs cultured in MSC-CM produce IGF-II, which compensates for its absence in the MSC-CM.

\section{Materials and Methods}

\section{hESC maintenance}

Human ESC lines HS181 and SHEF1 (kindly provided by Prof. Outi Hovatta, Karolinska Institute, Sweden and Prof. Harry Moore and Peter Andrews, University of Sheffield, UK, respectively) were maintained in a feeder-free culture over matrigel-coated T25 flasks (BD Biosciences) in MSC-CM or HFF-CM. The basal media used to prepare the CM consisted of $80 \%$ KO-DMEM supplemented with $20 \%$ KO Serum Replacement, 1\% non-essential amino acids, $1 \mathrm{mM}$ L-glutamine, $0.1 \mathrm{mM} \beta$-mercaptoethanol and $8 \mathrm{ng} / \mathrm{ml}$ of bFGF (all from Invitrogen, CA). MSC-CM and HFF$\mathrm{CM}$ were prepared and collected as described in detail for MEFCM $[11,12]$. HFFs were purchased from ATCC (SCD-1112SK). Human MSCs were obtained from post-natal adipose tissue from healthy donors upon informed consent as described previously
$[27,28]$. During routine maintenance, HFFs and MSCs were grown in IMDM and advanced-DMEM, respectively, plus $10 \%$ FCS and $2 \mathrm{mM}$ L-glutamine and split in the ratio 1:2 when they reached $80-90 \%$ confluence. MSCs were fully characterized and showed typical fibroblast-like morphology, immunophenotype $\left(\mathrm{CD} 44^{+} \mathrm{CD} 90^{+} \mathrm{CD} 3^{+} \mathrm{CD} 105^{+} \mathrm{CD} 45^{\circ} \mathrm{CD} 34^{-} \mathrm{CD} 14^{-} \mathrm{HLA}^{-D R}{ }^{-}\right)$and in vitro differentiation capacity into osteoblasts, adipocytes and chondrocytes [7, 27-29]. Both hESC lines were maintained feederfree for 23-37 passages and were fed with MSC-CM or HFF-CM. The media was changed daily and the hESC cultures were split $(1: 2)$ weekly using collagenase IV $[10,12]$.

\section{Immunocytochemistry and flow-cytometric characterization of $h E S C S$}

Human ESC lines were phenotypically characterized by immunocytochemistry and flow cytometry for SSEA-3, SSEA-4 (both from DSHB, Iowa), and Tra-1-81 and TRA-1-60 (both from Chemicon, CA). For immunocytochemistry, hESC colonies were fixed in $4 \%$ of paraformaldehyde for $20 \mathrm{~min}$, followed by 30 min incubation in $10 \%$ normal goat serum in PBS. Colonies were incubated with primary antibodies (1:100 dilution) for $1 \mathrm{~h}$ at RT. Conjugated secondary antibodies (1:100) were used for $30 \mathrm{~min}$ at RT as follows: goat anti-rat IgG AMCA-conjugated for SSEA-4 and goat anti-mouse IgM-FITC conjugated for SSEA-3, TRA-1-60 and Tra-1-81. The slides were mounted in Vectashield containing DAPI. An irrelevant monoclonal antibody of the same isotype was used as a negative control.

For flow cytometry, collagenase IV-dissociated hESCs were suspended in PBS $+3 \%$ FBS at a concentration of $2-5 \times 10^{4}$ cells per $100 \mu \mathrm{l}$ and incubated with the specific primary antibody for 30 min at $4{ }^{\circ} \mathrm{C}$. After being washed, cells were incubated with $2.5 \mu \mathrm{l}$ of FITC-conjugated goat anti-mouse IgG antibody (Immunotech, Marseille, France). After $30 \mathrm{~min}$ at $4{ }^{\circ} \mathrm{C}$, stained cells were washed and stained with 7-aminoactinomycin D (7-AAD) viability dye (Immunotech) for $15 \mathrm{~min}$ at RT. Live cells identified by 7-AAD exclusion were analyzed for expression of SSEA-3, SSEA-4, Tra1-60 and Tra-1-81 using a FACSCanto-II flow cytometer equipped with the FACSDiva software (BDB) [30].

\section{RNA extraction, cDNA synthesis and RT-PCR detection of hESC markers and FGFRs}

Total RNA from hESCs, HFFs and MSCs was obtained using Trizol Reagent (Invitrogen). The first-strand cDNA was synthesized with $10 \mu$ of total RNA, using the High-Capacity cDNA Reverse Transcription Kit (Applied Biosystems). The samples were incubated at $25^{\circ} \mathrm{C}$ for $10 \mathrm{~min}$, followed by 2 -h incubation at $37^{\circ} \mathrm{C}$. The reaction was stopped at $85^{\circ} \mathrm{C}$ for $5 \mathrm{~s}$.

Expression of the pluripotency-associated transcription factors Oct3/4, Nanog, Sox-2 and Rex-1, as well as FGFR1-4, was assessed by end-point RT-PCR. GAPDH or ABL was used as the housekeeping gene. The primers used for the PCR were as follows: Oct3/4: 5'-TCT GCA GAA AGA ACT CGA GCA A-3' and 5'-AGA TGG TCG TTT GGC TGA ACA C-3'; Nanog: 5'-TGC AGT TCC AGC CAA ATT CTC-3' and 5'-CCT AGT GGT CTG CTG TAT TAC ATT AAG G-3'; Sox-2: 5'-CCC CCG GCG GCA ATA GCA-3' and 5'-TCG GCG CCG GGG AGA TAC AT-3'; Rex1: 5'-CAG ATC CTA AAC AGC TCG CAG AAT-3' and 5'-GCG TAC GCA AAT TAA AGT CCA GA-3'; FGFR1: 5'-GGA CTC TCC CAT CAC TCT GCA T-3' and 5'- CCC CTG TGC AAT AGA 
TGA TGA TC-3'; FGFR2: 5'-ACG TGG AAA AGA ACG GCA GTA-3' and 5'- AGC CAG CAC TTC TGC ATT GG-3'; FGFR3: 5'-AGG ATG CCT GCA TAC ACA CTG C-3' and 5'- ACA CCC TAC GTT ACC GTG CTC AAG-3'; FGFR4: 5'-GAA CCG CAT TGG AGG CAT T-3' and 5'-TTC TCT ACC AGG CAG GTG TAT GTG-3'; and GAPDH: 5'-GAA GGT GAA GGT CGG AGT C-3' and 5'-GAA GAT GGT GAT GGG ATT TC-3'. For Oct3/4, Nanog, Sox-2 and Rex-1, the conditions used for end-point PCR and QPCR were as follows: end-point PCR [5 min at $94{ }^{\circ} \mathrm{C}, 35$ cycles of $30 \mathrm{~s}$ at $94{ }^{\circ} \mathrm{C}$ followed by $50 \mathrm{~s}$ at $60{ }^{\circ} \mathrm{C}$ and $50 \mathrm{~s}$ at $72{ }^{\circ} \mathrm{C}$ and a final extension of $10 \mathrm{~min}$ at $72{ }^{\circ} \mathrm{C}$ ] and Q-PCR $\left[10 \mathrm{~min}\right.$ at $95^{\circ} \mathrm{C}$, 40 cycles of $30 \mathrm{~s}$ at $95^{\circ} \mathrm{C}$ followed by $60 \mathrm{~s}$ at $55^{\circ} \mathrm{C}$ and $60 \mathrm{~s}$ at 72 $\left.{ }^{\circ} \mathrm{C}\right]$. For the FGFRs the following PCR conditions were used: 2 min at $50{ }^{\circ} \mathrm{C}, 10 \mathrm{~min}$ at $95{ }^{\circ} \mathrm{C}, 40$ cycles of $15 \mathrm{~s}$ at $95{ }^{\circ} \mathrm{C}$ followed by $60 \mathrm{~s}$ at $60^{\circ} \mathrm{C}$.

\section{Conventional karyotyping}

G-banding studies were performed as described previously [3134]. Briefly, hESC lines were cultured in hESC medium supplemented with $0.1 \mathrm{mg} / \mathrm{ml}$ colcemid (Biological Industries) for up to 3-4 h. The cells were then washed in Versene solution (Gibco) and subsequently trypsinized and spun down. The pellet was resuspended carefully in a $\mathrm{KCl}$ hypotonic solution $(0.075 \mathrm{~mol} / \mathrm{L})$, rinsed to remove the cytoplasm, and then fixed in methanol/acetic acid 3:1. The fixing procedure was repeated three times. Finally, the pellet was resuspended in a final volume of $1 \mathrm{ml}$ of fixative, and the cells were dropped onto glass slides. Chromosomes were visualized by using a modified Wright's staining. Twenty metaphases were analyzed for each cell line using a conventional microscope and the IKAROS-software (Metasystems, GmbH, Altlussheim, Germany).

\section{Embryoid body (EB) formation and in vitro differentiation}

Human ESCs were harvested, transferred to low-attachment plates and allowed to differentiate spontaneously through EB formation in KO-DMEM supplemented with $20 \%$ FCS (Invitrogen), $1 \%$ L-glutamine, $0.1 \mathrm{mM}$ non-essential amino acids and $0.1 \mathrm{mM}$ $\beta$-mercaptoethanol, but without bFGF, with media changes every 4 days. After 21 days of differentiation, EBs were spun down, fixed with $4 \%$ paraformaldehyde for $10 \mathrm{~min}$ and embedded in paraffin [31]. For each staining, three sections per specimen were used. The EB cells were incubated ( $1 \mathrm{~h}$ at RT) with primary antibodies for anti- $\alpha$-fetoprotein, anti- $\beta$ III-tubulin and anti-smooth muscle actin (Chemicon, 1:100). Subsequently, the sections were incubated with a biotinylated secondary antibody ( $30 \mathrm{~min}$ at RT) and a streptavidin peroxidase complex (30 min at RT) (Vector Laboratories Inc). The immunostaining was visualized using diaminobenzidine and counterstained with hematoxylin. The washing steps were done in PBS. Negative controls were prepared by replacing the primary antibody by PBS.

\section{In vivo teratoma formation}

Animal protocols were approved by the Local University Hospital Council On Animal Care and Experimentation. In vivo pluripotency was tested as described previously [31, 33, 34]. In brief, HS181 and SHEF1 hESCs were harvested with Collagenase IV. Human ESCs were implanted beneath the testicular capsule of 6-8-week-old NOD/SCID IL2R $\gamma^{-/-}$male mice $(n=17)$ (The Jackson Lab, Bar Harbor, MA). Teratoma growth was determined by weekly palpation every week, and the mice were killed 7-10 weeks after implantation. Teratomas were fixed, embedded in paraffin, and sections were stained with hematoxylin and eosin.

\section{TGF- $\beta$ and IGF-II detection and FGFRI and IGF-II block- ing experiments}

To determine whether bFGF cooperates with TGF- $\beta$ and IGFII, MSCs and HFFs were plated and treated with or without $8 \mathrm{ng} /$ $\mathrm{ml}$ bFGF. The concentration of soluble TGF- $\beta$ released into the media was measured by ELISA (Human TGF- $\beta$ ELISA Set BD OptEIA $^{\mathrm{TM}}$; BD Biosciences). For soluble IGF-II detection, the human IGF-II ELISA Kit (Diagnostic System Laboratories, Inc., Webster, TX) was used as described previously [5]. The production of IGF-II in response to bFGF was also assessed at the RNA level by real-time Q-PCR. Q-PCR was performed with Quantitect ${ }^{\mathrm{TM}} \mathrm{SYBR}^{\circledR}$ Green PCR Master Mix (Qiagen, Valencia, CA) and analyzed with the Mx3005P real-time PCR system (Stratagene, La Jolla, CA). IGF-II primer sequences used were 5'-TTG CTC TAC CCA CCC AAG AC-3' and 5'-GAT GGA ACC TGA TGG AAA CG-3'. Real-time PCR conditions were as follows: $10 \mathrm{~min}$ at 95 ${ }^{\circ} \mathrm{C}, 40$ cycles of $30 \mathrm{~s}$ at $95{ }^{\circ} \mathrm{C}$ followed by $60 \mathrm{~s}$ at $60{ }^{\circ} \mathrm{C}$ and $30 \mathrm{~s}$ at $72^{\circ} \mathrm{C}$. IGF-II mRNA expression was normalized against GAPDH. Inhibition of bFGF signals has been previously demonstrated with the FGFR1-specific chemical inhibitor SU5402 [5]. Before adding bFGF to MSCs or HFFs cultures, FGFR1 was inhibited for 60 min with $20 \mu \mathrm{M}$ SU5402 (Calbiochem) or with an equivalent volume of DMSO as control. The media was harvested $24 \mathrm{~h}$ later and the levels of soluble TGF- $\beta$ were further quantified by ELISA. Interference with IGF-II signalling in hESC cultures was accomplished by specific ligand neutralization through addition of $2 \mu \mathrm{g} / \mathrm{ml}$ of the antibody $\alpha$-hIGF-II (R\&D Systems; AF-292-NA), as described previously [5]. As an appropriate control, an immunoglobulin isotype match was used. After 16-day treatment with the IGF-II neutralizing antibody, cell proliferation, cell death, hESC colony morphology, expression of hESC-associated surface markers and transcription factors were assessed.

\section{Acknowledgments}

This work was partially funded by The Andalusian Health Department (0027/2006 to JG-C, 0028/2006 and 0030/2006 to PM), Andalusian Science and Innovation Department (P08-CTS-3678 to PM), The Jose Carreras International Foundation against the Leukemia (FIJC-05/EDThomas 2006 to PM/CB), The Spanish Ministry of Health to PM (FIS PI070026) and to JG-C (FIS PI052217). CB is supported by the FIS-ISCIII (CP07/00059). We are indebted to Prof. Peter Andrews (University of Sheffield) and Prof. Outi Hovatta (Karolinska University) for provision of the hESC lines used in the present study. We also thank Dr Deborah Burks (CIPF, Valencia, Spain) for providing MCF-7 and Hela cell lines and for her insightful feedback and helpful discussions.

\section{References}

1 Thomson JA, Itskovitz-Eldor J, Shapiro SS, et al. Embryonic stem cell lines derived from human blastocysts. Science 1998; 282:1145-1147.

2 Keller G. Embryonic stem cell differentiation: emergence of a new era in biology and medicine. Genes Dev 2005; 19:11291155 . 
3 Menendez P, Bueno C, Wang L. Human embryonic stem cells: a journey beyond cell replacement therapies. Cytotherapy 2006; 8:530-541.

4 Przyborski SA. Differentiation of human embryonic stem cells after transplantation in immune-deficient mice. Stem Cells 2005; 23:1242-1250.

5 Bendall SC, Stewart MH, Menendez P, et al. IGF and FGF cooperatively establish the regulatory stem cell niche of pluripotent human cells in vitro. Nature 2007; 448:1015-1021.

6 Scadden DT. The stem-cell niche as an entity of action. Nature 2006; 441:1075-1079.

7 Cobo F, Navarro JM, Herrera MI, et al. Electron microscopy reveals the presence of viruses in mouse embryonic fibroblasts but neither in human embryonic fibroblasts nor in human mesenchymal cells used for hESC maintenance: toward an implementation of microbiological quality assurance program in stem cell banks. Cloning Stem Cells 2008; 10:65-74.

8 Hovatta O, Mikkola M, Gertow K, et al. A culture system using human foreskin fibroblasts as feeder cells allows production of human embryonic stem cells. Hum Reprod 2003; 18:1404-1409.

9 Cheng L, Hammond H, Ye Z, et al. Human adult marrow cells support prolonged expansion of human embryonic stem cells in culture. Stem Cells 2003; 21:131-142.

10 Menendez P, Wang L, Chadwick K, et al. Retroviral transduction of hematopoietic cells differentiated from human embryonic stem cell-derived CD45(neg)PFV hemogenic precursors. Mol Ther 2004; 10:1109-1120.

$11 \mathrm{Xu} \mathrm{C}$, Inokuma MS, Denham J, et al. Feeder-free growth of undifferentiated human embryonic stem cells. Nat Biotechnol 2001; 19:971-974.

12 Wang L, Li L, Menendez P, et al. Human embryonic stem cells maintained in the absence of mouse embryonic fibroblasts or conditioned media are capable of hematopoietic development. Blood 2005; 105:4598-4603.

13 Stewart MH, Bendall SC, Bhatia M. Deconstructing human embryonic stem cell cultures: niche regulation of self-renewal and pluripotency. J Cell Mol Med 2008; 86:875-886.

14 Bigdeli N, Andersson M, Strehl R, et al. Adaptation of human embryonic stem cells to feeder-free and matrix-free culture conditions directly on plastic surfaces. J Biotechnol 2008; 133:146-153.

15 James D, Levine AJ, Besser D, et al. TGFbeta/activin/nodal signaling is necessary for the maintenance of pluripotency in human embryonic stem cells. Stem Cells Dev 2005; 132:1273-1282.

16 Smith JR, Vallier L, Lupo G, et al. Inhibition of Activin/Nodal signaling promotes specification of human embryonic stem cells into neuroectoderm. Dev Biol 2008; 313:107-117.

17 Vallier L, Reynolds D, Pedersen RA. Nodal inhibits differentiation of human embryonic stem cells along the neuroectodermal default pathway. Stem Cells Dev 2004; 275:403-421.

18 Vallier L, Alexander M, Pedersen RA. Activin/Nodal and FGF pathways cooperate to maintain pluripotency of human embryonic stem cells. J Cell Sci 2005; 118:4495-4509.

19 Amit M, Margulets V, Segev H, et al. Human feeder layers for human embryonic stem cells. Biol Reprod 2003; 68:2150-
2156.

20 Eiselleova L, Peterkova I, Neradil J, et al. Comparative study of mouse and human feeder cells for human embryonic stem cells. Int J Dev Biol 2008; 52:353-363.

21 Ellerstrom C, Strehl R, Moya K, et al. Derivation of a xenofree human embryonic stem cell line. Stem Cells 2006; 24:2170-2176.

22 Inzunza J, Gertow K, Stromberg MA, et al. Derivation of human embryonic stem cell lines in serum replacement medium using postnatal human fibroblasts as feeder cells. Stem Cells 2005; 23:544-549.

23 Richards M, Fong CY, Chan WK, et al. Human feeders support prolonged undifferentiated growth of human inner cell masses and embryonic stem cells. Nat Biotechnol 2002; 20:933-936.

24 Richards M, Tan S, Fong CY, et al. Comparative evaluation of various human feeders for prolonged undifferentiated growth of human embryonic stem cells. Stem Cells 2003; 21:546-556.

25 Huang S, Terstappen LW. Formation of haematopoietic microenvironment and haematopoietic stem cells from single human bone marrow stem cells. Nature 1992; 360:745-749.

26 Lighten AD, Hardy K, Winston RM, et al. Expression of mRNA for the insulin-like growth factors and their receptors in human preimplantation embryos. Mol Reprod Dev 1997; 47:134-139.

27 Rubio D, Garcia S, Paz M, et al. Molecular characterization of spontaneous mesenchymal stem cell transformation. PloS ONE 2008; 3:e1398.

28 Rubio D, Garcia-Castro J, Martin MC, et al. Spontaneous human adult stem cell transformation. Cancer Res 2005; 65:3035-3039.

29 Garcia-Castro J, Trigueros C, Madrenas J, et al. Mesenchymal stem cells and their use as a cell replacement therapy and disease modelling tool. J Cell Mol Med 2008; 12:1-14.

30 Bueno C, Montes R, Martin L, et al. NG2 antigen is expressed in $\mathrm{CD} 34^{+} \mathrm{HPCs}$ and plasmacytoid dendritic cell precursors: is NG2 expression in leukemia dependent on the target cell where leukemogenesis is triggered? Leukemia 2008; 22:14751478.

31 Catalina P, Bueno C, Montes R, et al. Genetic stability of human embryonic stem cells: a first-step toward the development of potential hESC-based systems for modelling childhood leukemia. Leukemia Res 2008; doi:10.1016/j.leukres.2008.08.028.

32 Catalina P, Cobo F, Cortes JL, et al. Conventional and molecular cytogenetic diagnostic methods in stem cell research: a concise review. Cell Biol Int 2007; 31:861-869.

33 Cortes JL, Sanchez L, Catalina P, et al. Whole-blastocyst culture followed by laser drilling technology enhances the efficiency of inner cell mass isolation and embryonic stem cell derivation from good- and poor-quality mouse embryos: new insights for derivation of human embryonic stem cell lines. Stem Cells Dev 2008; 17:255-267.

34 Catalina P, Montes R, Ligero G, et al. Human ESCs predisposition to karyotypic instability: Is a matter of culture adaptation or differential vulnerability among hESC lines due to inherent properties? Mol Cancer 2008; 7:76-81. 